\title{
Case Series; Experience of the Management of 2 Patients with Severe Anorexia Nervosa in Sligo University Hospital using the MARSIPAN protocol.
}

Saolta

Grupa ollscoile cüram slainte

Hugh $\mathrm{CM}^{1}$, Harron, $\mathrm{MK}^{2}$, OConnor, $\mathrm{P}^{1}$, Kilcullen, $\mathrm{A}^{1}$, Toolan, $\mathrm{A}^{1}$, Burns, $\mathrm{N}^{1}$, OMahony, $E^{2}$.

${ }^{1}$ Department of Medicine, Sligo University Hospital, Sligo, Ireland.

${ }^{2}$ Sligo/Leitrim Mental Health Services, St Columbas Hospital, Sligo, Ireland.

\section{INTRODUCTION}

Anorexia nervosa has the highest mortality of all psychiatric conditions. In excess of $20 \%$ never recover and develop a chronic eating disorder ${ }^{2}$. In Ireland 200,000 individuals are formally diagnosed with Eating Disorder, 1.6 million in the UK. In Ireland 400 new cases are diagnosed annually ${ }^{3}$.

Mortality rates with Anorexia Nervosa range from $5 \%$ to $20 \%$. Most die of suicide, malnutrition but many die from refeeding syndrome or its complications.

Refeeding syndrome is under-recognized and undertreated in general medical wards.

Sligo University Hospital (SUH) uses the MARSIPAN protocol for the (Management of Really Sick Patients with Anorexia Nervosa) ${ }^{3}$ for inpatient treatment.

\section{PROTOCOL}

Admission to dedicated bed in the observation bay on designated medical ward.

- One-to-one supervision by trained health care assistant $24 / 7$ with instructions not to engage in superfluous conversation and watch for sabotage.

- Total bed rest

- 1 litre fluid restriction at outset.

- No bathroom visits (commode by bedside)

- No napkins to avoid food caching

- No phones, laptops, books and magazines to avoid

flicking/microexercising

- Closed windows

- Restriction of visitors

- No food brought in from outside, controlled food diary.

\section{SABOTAGING BEHAVIOUR}

- Micro-exercising with phones, laptops, books, magazine, movements of arms and legs while weaning off ventilator.

- Food caching

- large clothing/hair accessories

- Requests for open windows (in order to shiver and lose weight)

- "Toeing" (grabbing and pushing down on weighing scales with toes)

- Intentional retaining of bladder and bowel for weighing.

\section{CASE 1}

\section{CASE 2}

A 22 year-old lady presented with a chronic severe enduring eating disorder having been previously treated in a psychiatric institution twice for this condition. She had developed an ED at 16 years of age.

On this occasion she admitted voluntarily and reported that food and bowels were dominating her life. She lived alone, did not leave the house and her brother reported that the cupboards in the house contained only "Fortisip" nutritional supplements and laxatives. She had only eaten a handful of grapes in the last week. She had a BMI of $12.06 \mathrm{~kg} / \mathrm{m}^{2}$, BP 90/56mmhg, HR 52 bpm, Blood glucose 5.1 .

She was commenced on a naso-gastric feed as per protocol. Electrolyte supplementation was required for refeeding syndrome. Although weak she displayed significant sabotaging behaviour, microexercising in bed, on her phone/computer. She continued to hold bowel and bladder for weighing. She remained in hospital for 63 days with gradual reintroduction of oral diet and exercise.

9 months post discharge she has a BMI $18.5 \mathrm{kgs} / \mathrm{m}^{2}$ and a part-time job. She reports that it is still difficult and she thinks about food a lot but is coping better.
27 year old lady presented with $10 \mathrm{lbs}$ weight loss in 3 weeks eating only celery, and a BMI $13.0 \mathrm{kgs} / \mathrm{m}^{2}$. The MARSIPAN protocol was commenced as described, allowing only vitamin supplementation orally.

On day 3 she became hypoglyaemic $(2.6 \mathrm{mmol} / \mathrm{L})$, dyspnoeic and desaturated to $85 \%$ despite $100 \%$ oxygen. Clinically and radiographcally she had developed an aspiration pneumonia. Respiratory failure required mechanical ventilation and inotropic support and electrolyte supplementation for refeeding syndrome. Upon weaning sedation she commenced micro-exercising with limb movements, animated conversations, requests for opening of windows to shiver and lose calories. She remained in hospital for 83 days. 10 months post discharge she has a BMI of $20 \mathrm{~kg} / \mathrm{m}^{2}$ and is living independently.

\section{DISCUSSION}

This is a very challenging cohort of patients with a significant mortality. Close electrolyte and cardiac monitoring is important for the first 10 days of refeeding. Sabotaging behaviour is inventive and relentless and teams need to be vigilant, especially at night when young interns may chart laxatives, kitchen staff change over and any number of potential sabotaging opportunities arise.

The nature of the illness crosses community/hospital and medical/nursing/psychiatric domains and as such is prone to falling between stools. Similarly it doesn't come into any medical specialty and refeeding syndrome is poorly recognised and treated on general medical wards.

Close collaboration and dogged persistance are the keys to success with this cohort of patients. Physicians need to be upskilled in the identification and management of eating disorders to avoid this thoroughly unavoidable deaths in young women

\section{References}

1. STEINHAUSEN, H. C. (2002) The
outcome of anorexia nervosa in the
20th century. Am J Psychiatry, 159,
1284-1293.
2. Dept of Health and Children, 2014,
Ireland.
www.welfare.ie/en/downloads/protoc
ol9.pdf
3. THE ROYAL COLLEGES OF
PSYCHIATRISTS PHYSICIANS AND
PATHOLOGISTS. (2014)
MARSIPAN: Management of Really
Sick Patients with Anorexia Nervosa.

